\title{
Lepton flavor violating signals of the neutral top-pion in future lepton colliders
}

\author{
Chong-Xing Yue, Zheng-Jun Zong, Li Zhou, Shuo Yang \\ Department of Physics, Liaoning Normal University, Dalian, 116029. P. R. China * \\ May 9, 2021
}

\begin{abstract}
The presence of the top-pions $\pi_{t}^{0, \pm}$ in the low-energy spectrum is an inevitable feature of the topcolor scenario. Taking into account the constraints of the present experimental limit of the lepton flavor violating $(L F V)$ process $\mu \rightarrow e \gamma$ on the free parameters of topcolor-assisted techicolor(TC2) models, we study the contributions of the neutral top-pion $\pi_{t}^{0}$ to the $L F V$ processes $\mu^{+} \mu^{-} \rightarrow \tau \mu$ ( or $\tau e$ ), $\gamma \gamma \rightarrow \tau \mu$ (or $\tau e), e^{+} e^{-} \rightarrow \tau \mu$, and $e \gamma \rightarrow e \pi_{t}^{0} \rightarrow e \tau \mu(e)$ via the flavor changing $(F C)$ couplings $\pi_{t}^{0} l_{i} l_{j}$ and discuss the possibility of searching for the $L F V$ signals via these processes in future lepton colliders.
\end{abstract}

PACS number: 12.60.Cn, 13.35.Dx,14.70.Pw

*E-mail:cxyue@lnnu.edu.cn 


\section{Introduction}

The solar neutrino experiments[1] and the atmospheric neutrino experiments[2] confirmed by reactor and accelerator experiments[3] have made one believe that neutrinos are massive and oscillate in flavors, and provide the only direct observation of physics that cannot be accommodated within the standard model $(S M)$, which can be seen as the first experimental clue for the existence of new physics beyond the $S M$. Thus, the $S M$ requires some modification to account for the pattern of neutrino mixing, in which the lepton flavor violating $(L F V)$ processes are allowed. The observation of the $L F V$ signals in present or future high-energy experiments would be a clear signature of new physics beyond the $S M$.

Many kinds of popular specific models beyond the $S M$ predict the presence of new particles, such as new gauge bosons and new scalars, which can naturally lead to the tree-level $L F V$ couplings. In general, these new particles could enhance branching ratios for some $L F V$ processes, and perhaps bringing them into the observable threshold of the present and next generations of collider experiments. Furthermore, nonobservability of these $L F V$ processes can lead to strong constraints on the nature of new physics. Thus, studying the possible $L F V$ signals of new particles in various high-energy colliders is very interesting and needed.

To completely avoid the problems arising from the elementary Higgs field in the $S M$, various kinds of dynamical electroweak symmetry breaking ( $E W S B$ ) models have been proposed, and among which the topcolor scenario is attractive because it can explain the large top quark mass and provide possible EWSB mechanism[4]. Almost all of these kind of models propose that the scale of the gauge groups should be flavor nonuniversal. When one writes the nonuniversal interactions in the masseigen basis, it can induce the tree -level flavor changing $(F C)$ couplings, which can generate rich phenomenology.

The presence of the physical top-pions $\pi_{t}^{0, \pm}$ in the low-energy spectrum is an inevitable feature of the topcolor scenario, regardless of the dynamics responsible for $E W S B$ and

other quark masses[5]. One of the most interesting features of the top-pions $\pi_{t}^{0, \pm}$ is that they have large Yukawa couplings to the third-generation quarks and can induce 
the tree-level $F C$ couplings in quark sector and in the lepton sector. The obviously phenomenological implication of this feature is that the top-pions can be significant produced via some $F C$ processes, and their possible signatures might be observed in future hadron colliders[5,6] and lepton colliders[7,8]. Furthermore, the top-pions can generate large corrections to some observables related with the $F C$ couplings and give characteristic signatures at various high-energy colliders $[9,10]$. On the other hand, the top-pions can generate significant contributions to the $L F V$ processes, such as $l_{i} \rightarrow l_{j} \gamma, l_{i} \rightarrow l_{j} l_{k} l_{l}$ and $Z \rightarrow l_{i} l_{j}[11]$. The branching ratios for some of these processes can be enhanced to their current or future experimental bounds, which can give severe constraints on the free parameters of topcolor scenario.

Among the various $L F V$ processes that have been considered in the literature, the most fruitful ones are the radiative decays $\tau \rightarrow e \gamma, \tau \rightarrow \mu \gamma, \tau \rightarrow e \eta$, and $\mu \rightarrow e \gamma$, since their branching ratios are tested with high precision[12,13]. These processes usually provide the restrictive experimental bounds on the free parameters of the popular specific models beyond the $S M$. For example, the contributions of the Higgs bosons to these processes have been extensively studied in Refs. $[14,15]$. Taking into account the constraints of the experimental upper limits of these $L F V$ processes on the free parameters, the $L F V$ decays of the Higgs bosons and possible signals in the future high-energy collider experiments have been studied in Ref.[16].

In this paper, we will study the $L F V$ signals of the neutral top-point $\pi_{t}^{0}$ at the future various lepton colliders in the context of the topcolor-assisted technicolor $(T C 2)$ models[17] with free parameters being compatible with the most relevant data of $\mu$ radiative decay $\mu \rightarrow e \gamma$. In this work, we consider the contributions of the neutral top-pion $\pi_{t}^{0}$ to the $L F V$ processes $\mu^{+} \mu^{-} \rightarrow \tau \mu$ ( or $\left.\tau e\right), \gamma \gamma \rightarrow \tau \mu$ (or $\left.\tau e\right), e^{+} e^{-} \rightarrow \tau \mu$, and $e \gamma \rightarrow e \pi_{t}^{0} \rightarrow e \tau \mu(e)$ via the $F C$ couplings $\pi_{t}^{0} l_{i} l_{j}$ and discuss possibility of directly searching for the $L F V$ signals in the future lepton colliders via these processes. For completeness, we also include an estimation of the contributions of the new gauge boson $Z^{\prime}$ predicted by $T C 2$ models to these processes and compare them with those of the neutral top-pion $\pi_{t}^{0}$ 
This paper is organized as follows. Section II contains a short summary of the relevant coupling to ordinary particles and decay modes of neutral top-pion $\pi_{t}^{0}$ in TC2 models. Discussions of the constraints coming from the $L F V \tau$ and $\mu$ decays, the muon anomalous magnetic moment $a_{\mu}$, and other observables on the relevant free parameters are also given in this section. Section III, IV and V are devoted to the computation of the produce cross sections generated by $\pi_{t}^{0}$ exchange for the $L F V$ processes $\mu^{+} \mu^{-} \rightarrow \tau \mu(\tau e), \gamma \gamma \rightarrow \tau \mu(\tau e), e^{+} e^{-} \rightarrow \tau \mu(\tau e)$, and $e \gamma \rightarrow e \tau \mu(e \tau e)$, respectively. Some phenomenological analyses are also included. Our conclusions are given in Sec. VI.

\section{The $L F V$ coupling of $\pi_{t}^{0}$ to ordinary particles}

For $T C 2$ models [17], technicolor $(T C)$ interactions play a main role in breaking the electroweak symmetry. Topcolor interactions make small contributions to EWSB and give rise to the main part of the top quark mass, $(1-\varepsilon) m_{t}$, with the parameter $\varepsilon<<1$, Thus, there is the following relation:

$$
\nu_{\pi}^{2}+F_{t}^{2}=\nu_{w}^{2}
$$

where $\nu_{\pi}$ represents the contributions of $T C$ interactions to $E W S B, \nu_{w}=\nu / \sqrt{2} \simeq 174 \mathrm{GeV}$. Here $F_{t} \simeq 50 \mathrm{GeV}$ is the physical top-pion decay constant. This means that the masses of the $S M$ gauge bosons $W$ and $Z$ are given by absorbing the linear combination of the top-pions and technipions. The orthogonal combination of the top-pion and technipions remains unabsorbed and physical[18,19,20]. However, the absorbed Goldstone boson linear combination is mostly the technipions, while the physical combination is mostly the top-pions, which are usually called physical top-pions $\left(\pi_{t}^{0,{ }^{ \pm}}\right)$.

For TC2 models, the underlying interactions, topcolor interactions, are nonuniversal and therefore do not possess Glashow-Iliopoulos-Maiani $(G I M)$ mechanism. The nonuniversal gauge interactions result in the new $F C$ coupling vertices when one writes the interactions in the mass eigenbasis. Thus, the top-pions can induce the new $F C$ coupling vertices. The couplings of the neutral top-pion $\pi_{t}^{0}$ to ordinary fermions, which are related to our calculation, can be written as $[5,8,11,17]$ : 


$$
\begin{aligned}
& \frac{m_{t}}{\sqrt{2} F_{t}} \frac{\sqrt{\nu_{w}^{2}-F_{t}^{2}}}{\nu_{w}}\left[K_{U R}^{t t} K_{U L}^{t t^{*}} \bar{t} \gamma^{5} t \pi_{t}^{0}+\frac{m_{b}-m_{b}^{\prime}}{m_{t}} \bar{b} \gamma^{5} b \pi_{t}^{0}+K_{U R}^{t c} K_{U L}^{t t^{*}} \overline{t_{L}} c_{R} \pi_{t}^{0}\right] \\
& +\frac{m_{l}}{\sqrt{2} \nu_{w}} \bar{l} \gamma^{5} l \pi_{t}^{0}+\frac{m_{\tau}}{\sqrt{2} \nu_{w}} K_{\tau i} \bar{\tau} \gamma^{5} l_{i} \pi_{t}^{0},
\end{aligned}
$$

where $m_{b}^{\prime} \approx 0.1 \varepsilon m_{t}$ is the part of the bottom-quark mass generated by extended technicolor $(E T C)[9] . K_{U L}$ and $K_{U R}$ are rotation matrices that diagonalize the up-quark mass matrix $M_{U}$, i.e. $K_{U L}^{+} M_{U} K_{U R}=M_{U}^{\text {dia }}$. To yield a realistic form of the CabibboKobayashi-Maskawa $(C K M)$ matrix $V$, it has been shown that their values can be taken as [5]:

$$
K_{U L}^{t t} \approx 1, \quad K_{U R}^{t t}=1-\varepsilon, \quad K_{U R}^{t c} \leq \sqrt{2 \varepsilon-\varepsilon^{2}}
$$

In the following calculation, we will take $K_{U R}^{t c}=\sqrt{2 \varepsilon-\varepsilon^{2}}$ and take $\varepsilon$ as a free parameter, which is assumed to be in the range of $0.01-0.1[4,17] . \quad l=\tau, \mu$ or $e, l_{i}(\mathrm{i}=1,2)$ is the first(second)generation lepton $e(\mu)$, and $k_{\tau i}$ is the flavor mixing factor between the thirdand the first-or second- generation leptons. Certainly, there is also the $F C$ scalar coupling $\pi_{t}^{0} \mu e$. However, the topcolor interactions only contact with the third-generation fermions, and thus, the flavor mixing between the first- and second-generation fermions is very small, which can be ignored.

The limits on the top-pion mass $m_{\pi_{t}}$ might be obtained via studying its effects on various experimental observables. For example, considering the couplings of the neutral top-pion $\pi_{t}^{0}$ to bottom quark through instanton effects, Ref.[18] has shown that the process $b \rightarrow s \gamma, B-\bar{B}$ mixing and $D-\bar{D}$ mixing demand that the top-pions are likely to be light, with masses of the order of a few hundred $\mathrm{GeV}$. Since the negative top-pion corrections to the $Z \rightarrow b \bar{b}$ branching ratio $R_{b}$ become smaller when the top-pion is heavier, the electroweak precision measurement data of $R_{b}$ give rise to a certain lower bound on the top-pion mass. It was shown that the top-pion mass should not be lighter than the order of $1 \mathrm{TeV}$ to make TC2 models consistent with the electroweak precision measurement data[19]. Reference [20] restudied this problem and found that the top-pion mass $m_{\pi_{t}}$ is allowed to be in the range of a few hundred $G e V$ depending on the models. Thus, the 
value of the top-pion mass $m_{\pi_{t}}$ remains subject to large uncertainty[4]. In general, the top-pion mass $m_{\pi_{t}}$ is allowed to be in the range of a few hundred $G e V$ depending on the models. In this paper, we will assume $150 \mathrm{GeV} \leq m_{\pi_{t}} \leq 350 \mathrm{GeV}$. In this case, the possible decay modes of $\pi_{t}^{0}$ are $b \bar{b}, \bar{t} c, \bar{f} f(f$ is the first-or second-generation fermions, or the third-generation bottom quark or leptons), $g g, \gamma \gamma$ and $\tau l,(l=\mu$ or $e)$. The total decay width which has been calculated in Ref.[21]. However, the modes of decay into leptons are not included in this reference. If we take into account these decay modes, then the branching ratio $\operatorname{Br}\left(\pi_{t}^{0} \rightarrow \tau \mu\right)$ is in the range of $7.2 \times 10^{-3} k_{\tau \mu}^{2} \sim 2.5 \times 10^{-5} k_{\tau \mu}^{2}$ for $150 \mathrm{GeV} \leq m_{\pi_{t}} \leq 350 \mathrm{GeV}$ and $0.01 \leq \varepsilon \leq 0.1$.

Since the topcolor interactions only contact with the third- generation fermions and the flavor mixing between the first- and second-generation fermions is very small, the $B$ system observables cannot give significant constraints on the flavor mixing factor $k_{\tau i}$.

It is well known that the precision measurement of the muon anomalous magnetic moment $a_{\mu}$ is a sensitive test for the new physics beyond the $S M$. Comparing the new measurement value of $a_{\mu}$ with the present $S M$ prediction, there remains a tantalizing discrepancy $[22]$ :

$$
a_{\mu}^{e x p}-a_{\mu}^{S M}=(24.5 \pm 9) \times 10^{-10}
$$

If we assume that the observed deviation, as shown in Eq.(4), comes from the contributions of new particles predicted by $T C 2$ models, then we might obtain a constraint on $T C 2$ models using this deviation[23]. However, we find that the contributions of TC2 models to $a_{\mu}$ come mainly from the ETC gauge bosons and nonuniversal gauge boson $Z^{\prime}$; the contributions of $\pi_{t}^{0}$ are smaller than $1 \times 10^{-13}$ i.e. $\delta a_{\mu}^{\pi_{t}^{0}}<1 \times 10^{-13}$. Thus, the recently measurement value of $a_{\mu}$ can not give significant constraints on the free parameters, which are related to the top-pion, such as $\varepsilon, m_{\pi_{t}}$, and $k_{\tau_{i}}$.

The neutral top-pion $\pi_{t}^{0}$ can produce significant contributions to the $L F V$ processes $l_{i} \rightarrow l_{j} \gamma, l_{i} \rightarrow l_{j} l_{k} l_{l}$, and $Z \rightarrow l_{i} l_{j}$ via the $F C$ couplings $\pi_{t}^{0} \tau \mu$ and $\pi_{t}^{0} \tau e$, and can enhance the branching ratios of these processes by several orders of magnitude[11]. For the processes $l_{i} \rightarrow l_{j} \gamma$, the contributions come from the on-shell photon penguin diagrams, while the contributions come from both the on-shell photon penguin diagrams and the 
tree-level diagrams for the processes $\tau \rightarrow l_{i} l_{j} l_{k}$. Reference [11] has shown that, in all of the parameter space of TC2 models, the branching ratios of the processes $\tau \rightarrow l \gamma$, $l_{i} \rightarrow l_{j} l_{k} l_{l}$, and $Z \rightarrow l_{i} l_{j}$ are far below the experimental upper bound on these processes, except for the process $\mu \rightarrow 3 e$, which might approach the observable threshold of nearfuture experiments[13]. The present experimental limit of the process $\mu \rightarrow e \gamma$ can give severe constraints on the free parameters of TC2 models. If we assume $k=k_{\tau \mu}=k_{\tau e}$ and $150 \mathrm{GeV} \leq 350 \mathrm{GeV}$, then there must be $k \leq 0.16$. Taking into account this constraint, we will study possible $L F V$ signals of the neutral top-pion $\pi_{t}^{0}$ at future various lepton colliders in the following sections.

\section{LFV signals of the neutral top-pion $\pi_{t}^{0}$ in the future muon collders}

A muon collider is an excellent tool to study the properties of a heavy scalar or pseudoscalar and potential new physics effects[24]. It has been shown that a large number of Higgs bosons[24] or new particles, such as technihadron and technipions[25], can be produced through s-channel resonance processes. The $F C$ scalar couplings[26,27] might be tested at future muon colliders. Thus, the future muon collider opens up the possibility of studying the $L F V$ signals mediated by the new scalars.

The neutral top-pion $\pi_{t}^{0}$ has $F C$ couplings to the leptons at tree level and thus can contribute to the processes $\mu^{+} \mu^{-} \rightarrow \tau \mu(\tau e)$ via $\pi_{t}^{0}$ exchange in the s-channel. In spite of the fact that the coupling $\pi_{t}^{0} \mu^{+} \mu^{-}$, being proportional to $m_{\mu} / v$, is very small, if the muon collider with the center-of-mass $($ c.m. $)$ energy $\sqrt{s}$ runs on $\pi_{t}^{0}$ resonance $\left(\sqrt{s}=m_{\pi_{t}}\right)$, then the neutral top-pion $\pi_{t}^{0}$ may be produced at an appreciable rate[25, 27]. Thus, the neutral top-pion might give observable $L F V$ signals at future muon collider experiments.

After averaging over initial polarization and integrating over the scattering angle, it is straightforward to obtain the unpolarized cross section of the process $\mu^{+} \mu^{-} \rightarrow \tau \mu(\tau e)$ mediated by $\pi_{t}^{0}$ exchange. The s-channel resonance cross section for this process can be approximately written as:

$$
\sigma(\tau \mu(e)) \approx \frac{4 \pi}{m_{\pi_{t}}} \cdot \operatorname{Br}\left(\pi_{t}^{0} \rightarrow \mu^{+} \mu^{-}\right) \operatorname{Br}\left(\pi_{t}^{0} \rightarrow \tau \mu(e)\right)
$$

where $\operatorname{Br}\left(\pi_{t}^{0} \rightarrow \mu^{+} \mu^{-}\right)$and $\operatorname{Br}\left(\pi_{t}^{0} \rightarrow \tau \mu(e)\right)$ represent the branching ratios of $\pi_{t}^{0}$ decaying 
to $\mu^{+} \mu^{-}$and $\tau \mu(e)$, respectively. Observably, there is $\sigma(\tau \mu) \approx \sigma(\tau e)$ for $k_{\tau \mu}=k_{\tau e}$ and neglecting the final state masses, which is different from that generated by the Higgs bosons[26]. In that case, compared the production cross section $\sigma(\tau \mu)$, the cross section $\sigma(\tau e)$ is suppressed by a factor of $m_{e} / m_{\mu} \sim 1 / 200$.

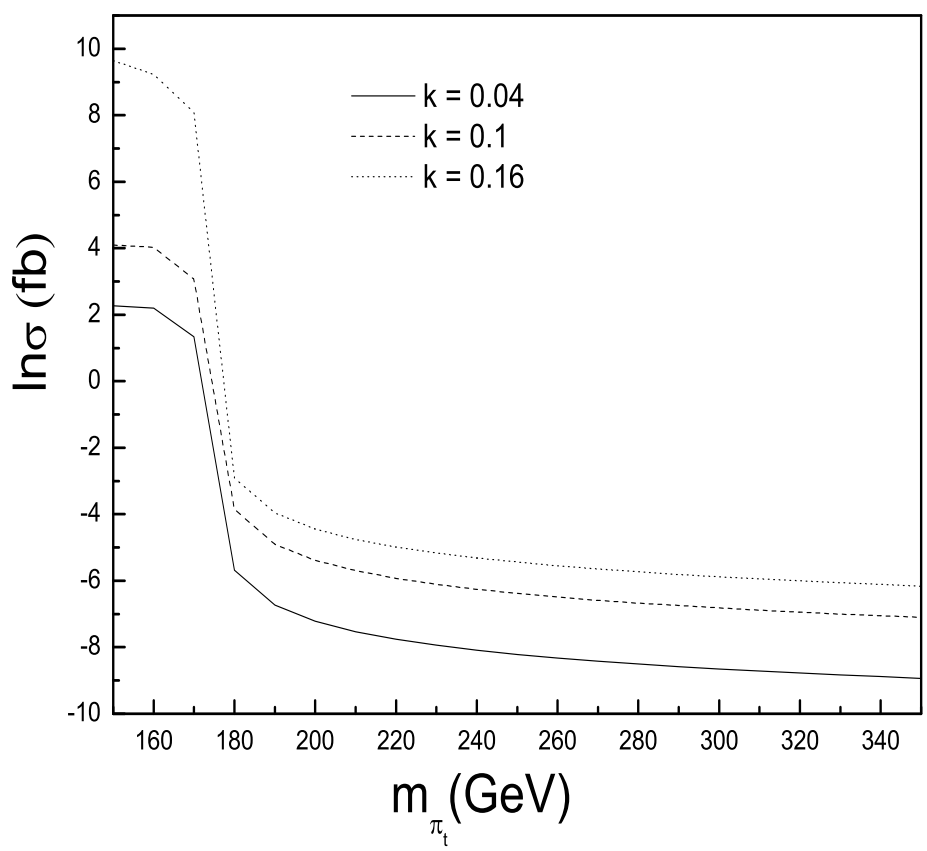

Figure 1: The resonance production cross section $\sigma(\tau \mu)$ as function of the top-pion mass $m_{\pi_{t}}$ for $\sqrt{s}=m_{\pi_{t}}$ and three values of the mixing parameter $k$.

In Fig.1 we show the resonance production cross section $\sigma(\tau \mu)$ as a function of the top-pion mass $m_{\pi_{t}}$ for $\sqrt{s}=m_{\pi_{t}}$ and three values of the mixing parameter $k$. Since the cross section $\sigma(\tau \mu)$ is not sensitive to the free parameter $\varepsilon$, we have taken $\varepsilon=0.05$ in Fig.1. One can see from Fig.1 that the $\sigma(\tau \mu)$ decreases with $m_{\pi_{t}}$ increasing and the mixing parameter $k$ decreasing. For $m_{\pi_{t}} \geq m_{t}+m_{c}$, the $F C$ channel $\pi_{t}^{0} \rightarrow \bar{t} c$ opens up and the branching ratios $\operatorname{Br}\left(\pi_{t}^{0} \rightarrow \mu^{+} \mu^{-}\right)$and $\operatorname{Br}\left(\pi_{t}^{0} \rightarrow \tau \mu\right)$ drop substantially, and thus the cross sections $\sigma(\tau \mu)$ and $\sigma(\tau e)$ drop considerably. The values of $\sigma(\tau \mu)$ are in the ranges of $1.54 \times 10^{2} \sim 3.4 \times 10^{-2} \mathrm{fb}$ and $5.5 \times 10^{-2} \sim 1.5 \times 10^{-4} \mathrm{fb}$ for $k \leq 0.16$, $150 \mathrm{GeV} \leq m_{\pi_{t}}<180 \mathrm{GeV}$, and $180 \mathrm{GeV} \leq m_{\pi_{t}} \leq 350 \mathrm{GeV}$, respectively. If we assume 
a future muon collider runing with the c.m. energy $\sqrt{s}=200 \sim 500 \mathrm{GeV}$ and a yearly integrated luminosity of $\mathcal{L}=20 \mathrm{fb}^{-1}$, then there will be several and up to thousand $\tau \mu$ (or $\tau e)$ events to be generated a year for $m_{\pi_{t}}<180 \mathrm{GeV}$.

For the $L F V$ processes $\mu^{+} \mu^{-} \rightarrow \tau \mu$ ( or $\tau e$ ), the final leptons always emerge back to back and carrying a constant energy which is one half of the c.m. energy $\sqrt{s}$. The main background would arise from the process $\mu^{+} \mu^{-} \rightarrow \bar{\tau} \mu \overline{\nu_{\mu}} \nu_{\tau}$ or $\bar{\tau} e \overline{\nu_{e}} \nu_{\tau}$. It has been shown that the contributions to the background come mainly from the diagrams with Higgs boson exchange[26]. The background cross section can only reach a peak around $m_{H}=130 \mathrm{GeV}$ and then drops quickly out of this range. Thus, as long as $150 \mathrm{GeV} \leq m_{\pi_{t}} \leq m_{t}+m_{c}$, the $L F V$ signals of the neutral top pion $\pi_{t}^{0}$ should be observed via the resonance processes $\mu^{+} \mu^{-} \rightarrow \tau \mu($ or $\tau e)$ in the future muon colliders.

TC2 models also predict the existence of a nonuniversal $U(1)$ gauge boson $Z^{\prime}$, which can lead to the tree-level $F C$ couplings $Z^{\prime} \tau \mu$ and $Z^{\prime} \tau e$. Thus, the nonuniversal gauge boson $Z^{\prime}$ has contributions to the $L F V$ processes $\mu^{+} \mu^{-} \rightarrow \tau \mu($ or $\tau e)$ via the s-channel $Z^{\prime}$ exchange. At leading order, the unpolarized cross section $\sigma^{\prime}(\tau \mu)$ generated by $Z^{\prime}$ exchange can be written as:

$$
\sigma^{\prime}(\tau \mu)=\frac{25 \pi^{2} \alpha^{2} \lambda_{\tau \mu}^{2}}{12 C_{W}^{6} K_{1}} \frac{s}{\left(s-M_{Z^{\prime}}^{2}\right)^{2}+M_{Z^{\prime}}^{2} \Gamma_{Z^{\prime}}^{2}},
$$

where $C_{W}=\cos \theta_{W}, \theta_{W}$ is the Weinberg angle, $\lambda_{\tau \mu}$ is the coupling constant of the $F C$ vertex $Z^{\prime} \tau \mu$. $K_{1}$ is the mixing parameter between the nonuniversal gauge boson $Z^{\prime}$ and the $S M$ gauge boson $Z$. Using Eq.(6), we can easily give the numerical results. However, considering the constraints of the electroweak precision measurement data on the $Z^{\prime}$ mass $M_{Z^{\prime}}\left(M_{Z^{\prime}}>1 T e V\right)[4,28]$, the cross section $\sigma^{\prime}(\tau \mu)$ is smaller than $10^{-4} f b$ in all of the parameter space at the future muon colliders with $\sqrt{s}=300 \sim 500 \mathrm{GeV}$, which cannot produce observable signals. Furthermore, even if the nonuniversal gauge boson $Z^{\prime}$ can produce observable $L F V$ signals, we can use polarized beams to separate the contributions from $\pi_{t}^{0}$ exchange from those from $Z^{\prime}$ exchange.

\section{The neutral top-pion $\pi_{t}^{0}$ and the $L F V$ processes $\gamma \gamma \rightarrow \pi_{t}^{0} \rightarrow \tau \mu(\tau e)$}

It is widely believed that hadron colliders, such as Tevatron and future $L H C$, can directly probe possible new physics beyond the $S M$ up to a few $T e V$, while the future 
high-energy linear $e^{+} e^{-}$collider $(I L C)$ is also required to complement the probe of the new particles with detailed measurement. A future $I L C$ will offer an excellent opportunity to study production and decay of the new particles with percent-level-precision[29]. A unique feature of the future $I L C$ is that it can be transformed to $\gamma \gamma$ colliders with the photon beams generated by the Compton backward scattering of the initial electron and laser beams. Their effective luminosity and energy are expected to be comparable to those of the $I L C$. In some scenarios, they are the best instrument for the discovery of signals of new physics[30].

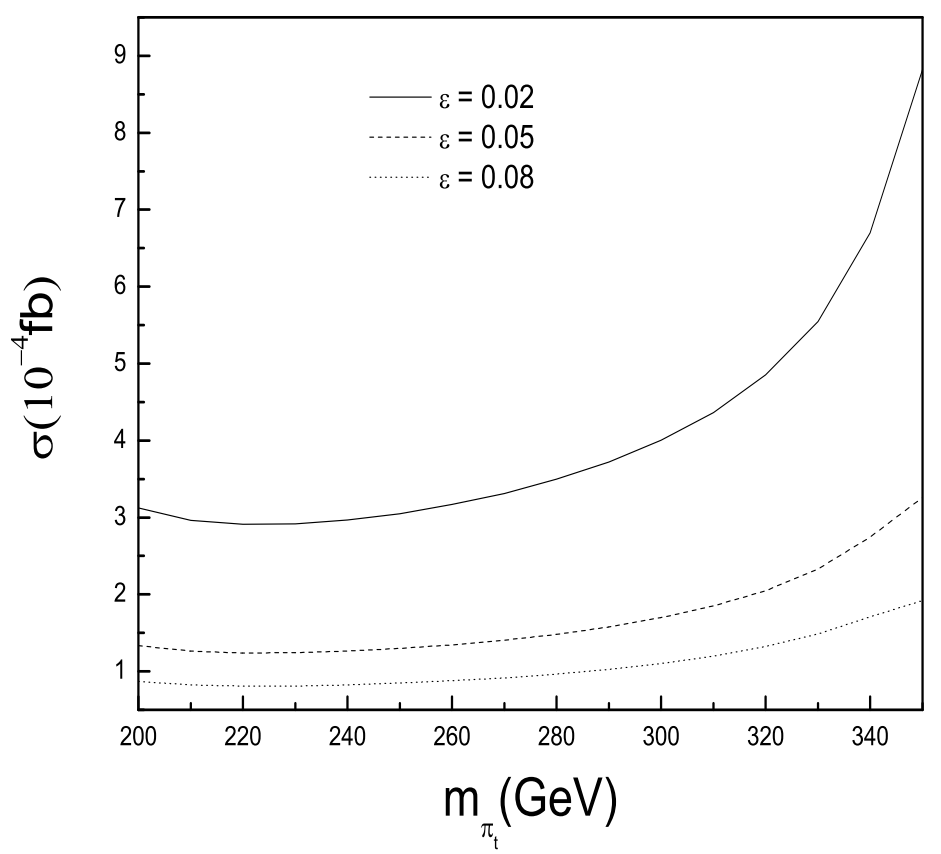

Figure 2: The effective cross section $\sigma(s)$ as a function of $m_{\pi_{t}}$ for $\sqrt{s}=500 \mathrm{GeV}, k=0.1$, and three values of the free parameter $\varepsilon$.

Similar to the contributions of the neutral top-pion $\pi_{t}^{0}$ to top-charm associated production at $\gamma \gamma$ colliders[7,9], the contributions of $\pi_{t}^{0}$ to the $L F V$ processes $\gamma \gamma \rightarrow \tau \mu(\tau e)$ via the $F C$ couplings $\pi_{t}^{0} l_{i} l_{j}$ proceed through the self-energy diagrams, vertex diagrams and the s-channel triangle diagram. We have explicitly calculated the contributions of these Feynman diagrams and found that the dominant contributions come from the s- 
channel triangle diagram, whereas the remaining diagrams give negligible contributions. Our numerical results are shown in Fig.2, in which we plot the effective cross section $\sigma(s)=\sigma\left(e^{+} e^{-} \rightarrow \gamma \gamma \rightarrow \tau \mu\right)$ for the $L F V$ process $\gamma \gamma \rightarrow \tau \mu$ as a function of the top-pion mass $m_{\pi_{t}}$ for the c.m. energy $\sqrt{s}=500 \mathrm{GeV}$, the flavor mixing factor $k=0.1$, and three values of the parameter $\varepsilon$. From this figure, one can see that the cross section $\sigma(s)$ for the process $e^{+} e^{-} \rightarrow \gamma \gamma \rightarrow \tau \mu$ (or $\tau e$ ) is relatively insensitive to the parameter $\varepsilon$. As the top-pion mass $m_{\pi_{t}}$ increases, the cross section increases monotonically. For $k=0.1$ and $200 \mathrm{GeV} \leq m_{\pi_{t}} \leq 350 \mathrm{GeV}$, the value of the cross section $\sigma\left(e^{+} e^{-} \rightarrow \gamma \gamma \rightarrow \tau \mu\right)$ is smaller than $1 \times 10^{-3} \mathrm{fb}$. Certainly, if we assume $150 \mathrm{GeV} \leq m_{\pi_{t}}<180 \mathrm{GeV}$, then the cross section increases rapidly and its value can reach $8.3 \times 10^{-2} \mathrm{fb}$.

The flavor mixing parameter $k$ controls the strength of the $F C$ coupling $\pi_{t}^{0} \tau l(l=\mu$ or e) and further determines the $L F V$ signals of the neutral top-pion $\pi_{t}^{0}$. Its value is severely constrained by the current experimental upper bound on the $L F V$ process $\mu \rightarrow e \gamma[11]$. There is $k \leq 0.16$ for $m_{\pi_{t}} \leq 350 \mathrm{GeV}$. Taking into account this constraint, we plot the effective cross section $\sigma(s)$ as a function of $k$ for $\sqrt{s}=500 \mathrm{GeV}, \varepsilon=0.05$, and three values of the top-pion mass $m_{\pi_{t}}$ in Fig.3.

From the above discussion, we can see that, if we assume $200 \mathrm{GeV} \leq m_{\pi_{t}}<350 \mathrm{GeV}$, then the effective cross section $\sigma(s)$ is smaller than $1 \times 10^{-3} \mathrm{fb}$ in most of the parameter space consistent with the constraint from the $L F V$ process $\mu \rightarrow e \gamma$. However, for $150 \mathrm{GeV} \leq m_{\pi_{t}}<180 \mathrm{GeV}, \sigma(s)$ is in the range of $1 \times 10^{-2} \mathrm{fb} \sim 8 \times 10^{-2} \mathrm{fb}$. In this case, there will be several tens $\tau \mu(e)$ events to be generated a year at the future $I L C$ with $\sqrt{s}=500 \mathrm{GeV}$ and the yearly integrated $\mathcal{L}_{\text {int }}=340 \mathrm{fb}^{-1}$, which might be observed in future $I L C$ experiments.

The neutral top-pion $\pi_{t}^{0}$ can also contribute to the $L F V$ processes $e^{+} e^{-} \rightarrow \tau \mu(\tau e)$ via the $F C$ couplings $\pi_{t}^{0} \tau \mu(e)$. The relevant Feynman diagrams are shown in Fig.4. Since the s-channel process $e^{+} e^{-} \rightarrow \gamma^{*}, Z^{*} \rightarrow \tau \mu$ is suppressed by the propagators of the intermediate photon or Z-boson in the future $I L C$ with high center-of-mass energy, the cross section of the process $e^{+} e^{-} \rightarrow \tau \mu$ generated by the neutral top-pion $\pi_{t}^{0}$ should be smaller than that of the process $e^{+} e^{-} \rightarrow \gamma \gamma \rightarrow \tau \mu$. We have confirmed this expectation 


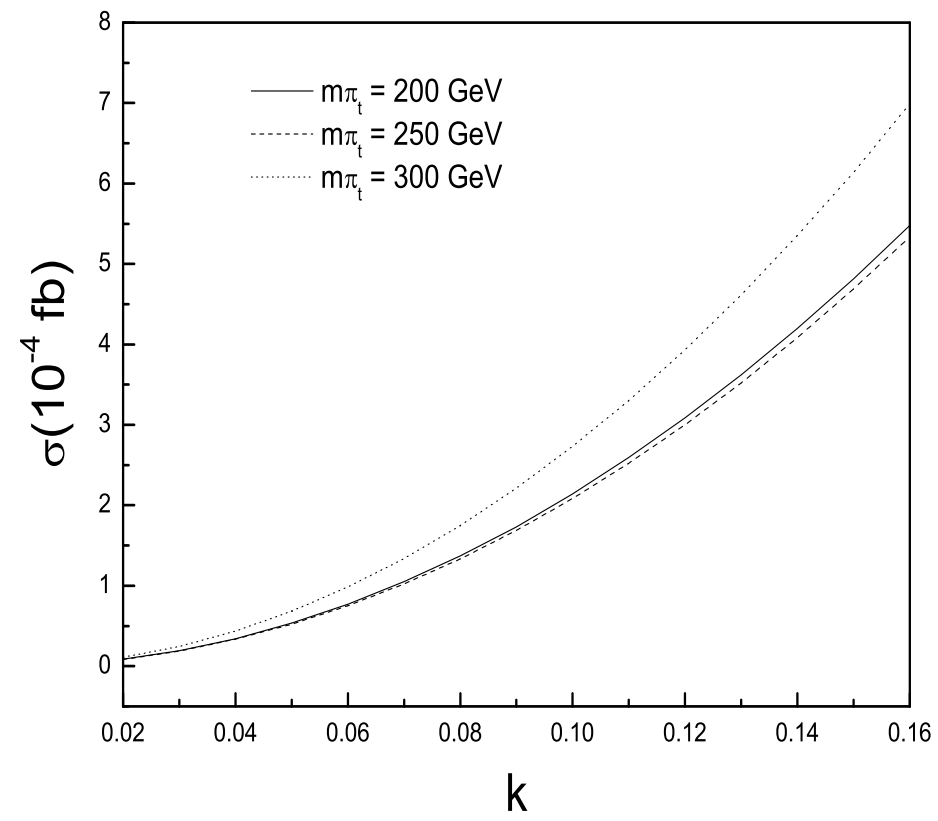

Figure 3: The effective cross section $\sigma(s)$ as a function of $k$ for $\sqrt{s}=500 \mathrm{GeV}, \varepsilon=0.05$ and three values of the $\pi_{t}^{0}$ mass $m_{\pi_{t}}$.

through explicit calculation. Our numerical results show that the cross section for the process $e^{+} e^{-} \rightarrow \tau \mu$ at the $I L C$ with $\sqrt{s}=500 \mathrm{GeV}$ is smaller than $1 \times 10^{-7} \mathrm{fb}$ in all of the parameter space of $T C 2$ models, which can not be detected in future $I L C$ experiments.

The production cross section of the process $e^{+} e^{-} \rightarrow Z^{\prime} \rightarrow \tau \mu(\tau e)$ is approximately equal to that of the process $\mu^{+} \mu^{-} \rightarrow Z^{\prime} \rightarrow \tau \mu(\tau e)$, which is smaller than $1 \times 10^{-4} f b$ in all of the parameter space of the TC2 models.

\section{The neutral top-pion $\pi_{t}^{0}$ and the $L F V$ process $e^{-} \gamma \rightarrow e^{-} \tau \mu$}

A future $I L C$ can also operate in $e^{-} \gamma$ collisions, where the $\gamma$ beam is generated by the Compton backward scattering of the incident position and laser beam and its energy and luminosity can reach the same order of magnitude of the corresponding position beam[30]. The $e^{-} \gamma$ collisions can produce particles which are kinematically not accessible at the $e^{+} e^{-}$ collisions at the same colliders. Thus, the $e^{-} \gamma$ collisions are well suite for studying the production and decays of new particles. In this section, we will discuss the contributions of the neutral top-pion $\pi_{t}^{0}$ to the $L F V$ process $e^{-} \gamma \rightarrow e^{-} \tau \mu$ and see whether the $L F V$ 


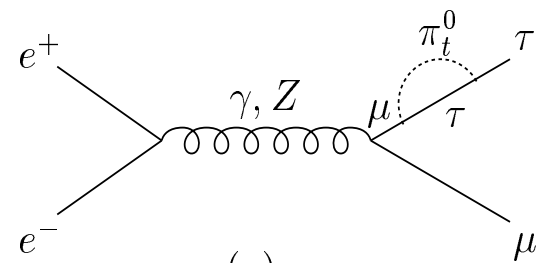

(a)

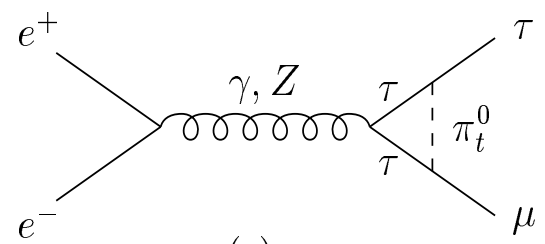

(c)

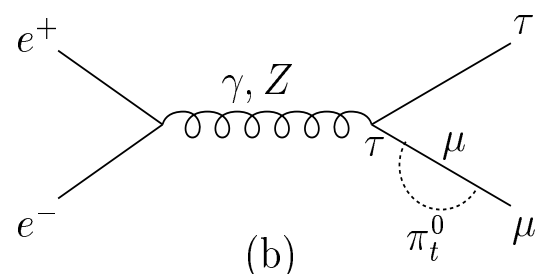

(b)

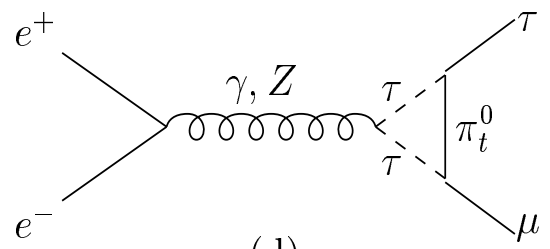

(d)

Figure 4: Feynman diagrams for the process $e^{+} e^{-} \rightarrow \tau \mu$ contributed by the $L F V$ coupling $\pi_{t}^{0} \tau \mu$

signals of $\pi_{t}^{0}$ can be detected via $e^{-} \gamma$ collisions in the future $I L C$.

In the TC2 models, $e^{-} \tau \mu$ production in $e^{-} \gamma$ collisions proceeds through the process $e^{-} \gamma \rightarrow e^{-} \gamma^{*} \gamma \rightarrow e^{-} \pi_{t}^{0} \rightarrow e^{-} \tau \mu$, in which the $\gamma$ beam is generated by the backward Compton scatting of incident positron and laser beam and the $\gamma^{*}$ beam is radiated from the $e^{-}$beam. The relevant Feynman diagram is shown in Fig.5. In our calculation, we use the Weizsäclcer - Williams approximation[31] and treat the virtual photon $\gamma^{*}$ coming from the $e^{-}$beam as a real photon. In this case, the effective cross section of the $L F V$ process $e^{-} \gamma \rightarrow e \tau \mu$ in the $I L C$ experiments can be obtained by folding the cross section of the subprocess $\gamma^{*} \gamma \rightarrow \tau \mu$ with the backscattered photon distribution function $F_{\gamma / e}(x)[32]$ and the function $P_{\gamma / e}\left(x, E_{e}\right)$, which is the probability of finding a photon with a fraction $x$ of energy $E_{e}$ in an ultrarelativistic electron[31].

Our numerical results are shown in Fig.6, in which we plot the cross section $\sigma(s)$ for the $L F V$ process $e^{+} e^{-} \rightarrow e^{-} \gamma \rightarrow e^{-} \gamma \gamma^{*} \rightarrow e^{-} \tau \mu$ as a function of $m_{\pi_{t}}$ for $\sqrt{s}=500 G e V$ and three values of the mixing parameter $k$. Since the cross section $\sigma(s)$ is not sensitive to the parameter $\varepsilon$, we have assumed $\varepsilon=0.05$ in Fig.6. One can see from Fig.6 that, for 


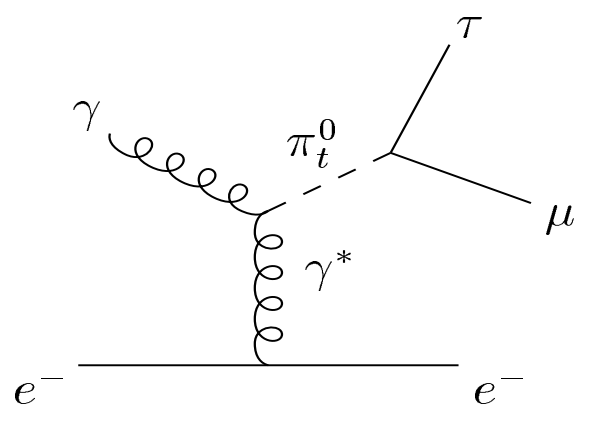

Figure 5: Feynman diagram for the process $e^{-} \gamma \rightarrow e^{-} \tau \mu$ contributed by the $L F V$ coupling $\pi_{t}^{0} \tau \mu$

$180 \mathrm{GeV} \leq m_{\pi_{t}} \leq 350 \mathrm{GeV}$, the value of the cross section $\sigma(s)$ is smaller than $4 \times 10^{-5} \mathrm{fb}$. Similar to the case for the process $e^{+} e^{-} \rightarrow \gamma \gamma \rightarrow \tau \mu$, the production cross section of this process for $150 \mathrm{GeV} \leq m_{\pi_{t}}<180 \mathrm{GeV}$ is significantly larger than that for $180 \mathrm{GeV} \leq$ $m_{\pi_{t}} \leq 350 \mathrm{GeV}$. For $150 \mathrm{GeV} \leq m_{\pi_{t}}<180 \mathrm{GeV}$, the effective cross section of the process $e^{-} \gamma \rightarrow e^{-} \tau \mu$ can reach $4.1 \times 10^{-3} f b$. Even in this case, i.e. $150 \mathrm{GeV} \leq m_{\pi_{t}}<180 \mathrm{GeV}$, the neuatral top-pion $\pi_{t}^{0}$ cannot produce observable signals via the $L F V$ process $e^{-} \gamma \rightarrow e^{-} \tau \mu$ in future lepton collider experiments.

\section{Conclusions}

The individual lepton numbers $L_{e}, L_{\mu}$, and $L_{\tau}$ are automatically conserved and the tree-level $L F V$ processes are absent in the $S M$, due to unitary of the leptonic analog of $C K M$ mixing matrix and the masslessness of the three neutrinos. However, the neutrino oscillation data provide very strong evidence for mixing and oscillation of the flavor neutrinos, which imply that the separated lepton numbers are not conserved. Thus, any observation of the effects for the $L F V$ processes would be a clear signature of new physics. This fact and the improvement of the relevant experimental measurements have brought considerable attention to study these processes in the context of specific popular models beyond the $S M$ and see whether the $L F V$ effects can be tested in future high-energy experiments. 


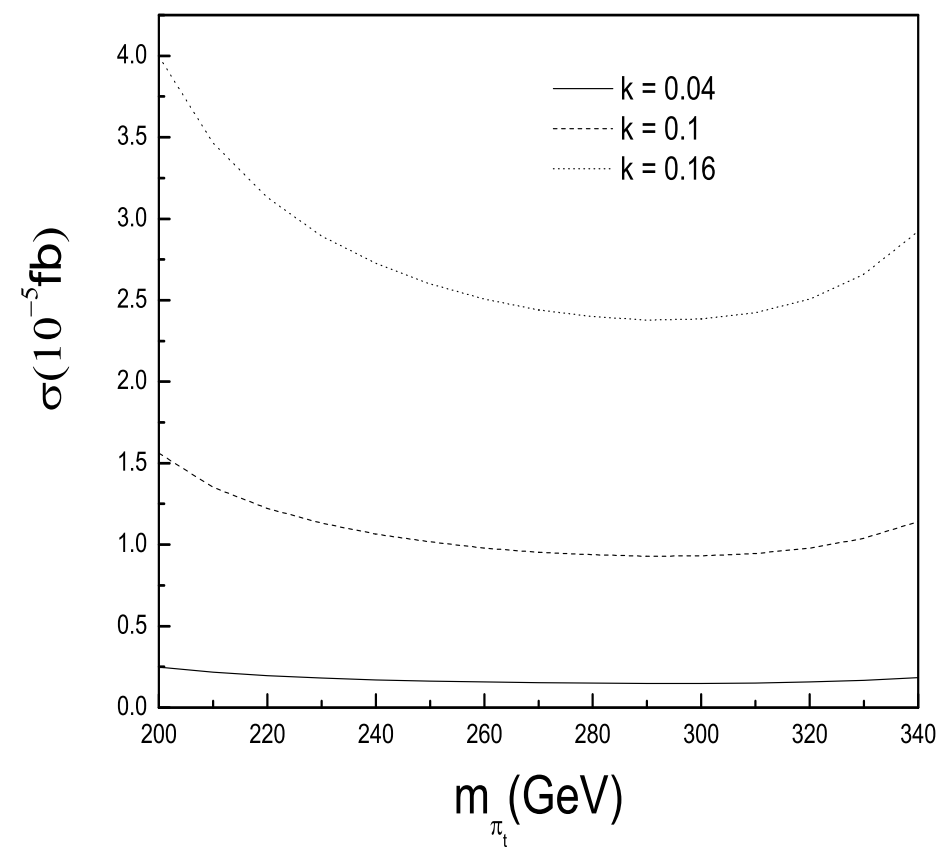

Figure 6: The effective cross section $\sigma(s)$ as a function of $m_{\pi_{t}}$ for $\sqrt{s}=500 \mathrm{GeV}, \varepsilon=0.05$ and three values of the mixing parameter $k$.

The topcolor scenario is one of the important candidates for the mechanism of $E W S B$. The presence of physical top-pions in the low-energy spectrum is a common feature of topcolor models. Since topcolor interactions are assumed to couple preferentially to the third generation and thus do not possess GIM mechanism, the physical top-pions have large Yukawa couplings to the third family fermions and can induce the $F C$ scalar couplings, which might give significant contributions to the $F C$ processes. The effects of the top-pion on these processes are governed by its mass $m_{\pi_{t}}$ and the relevant flavor mixing factors, which might produce observable signals at future high-energy experiments.

In this paper, we have calculated the contributions of the neutral top-pion $\pi_{t}^{0}$ predicted by TC2 models to the $L F V$ processes $\mu^{+} \mu^{-} \rightarrow \tau \mu(\tau e), \gamma \gamma \rightarrow \tau \mu(\tau e), e^{+} e^{-} \rightarrow \tau \mu(\tau e)$, and $e \gamma \rightarrow e \tau \mu(e \tau e)$, and discussed its possible $L F V$ signals in the future lepton colliders. We find that the value of the cross sections for these $L F V$ processes can indeed be enhanced by several orders of magnitude. With reasonable values of the free parameter, some of these processes may be within the observable threshold of near-future lepton collider 
experiments. For example, taking into account the constrains of the present experimental limit of the $L F V$ process $\mu \rightarrow e \gamma$ on the mixing factor $k$ and assuming $150 \mathrm{GeV} \leq$ $m_{\pi_{t}}<180 \mathrm{GeV}$, we find that the cross sections of the $L F V$ processes $\mu^{+} \mu^{-} \rightarrow \tau \mu$ and $e^{+} e^{-} \rightarrow \gamma \gamma \rightarrow \tau \mu$ can reach $1.5 \times 10^{2} f b$ and $8.3 \times 10^{-2} f b$, respectively. However, for $m_{\pi_{t}} \geq 200 \mathrm{GeV}$, the cross sections of all of the $L F V$ processes are very small, which cannot be detected in future experiments. Thus, we expect that the light top-pions predicted by topcolor scenario might produce observable $L F V$ signals in future lepton collider experiments.

\section{ACKNOWLEDGMENTS}

This work was supported in part by Program for New Century Excellent Talents in University(NCET-04-0209), the National Natural Science Foundation of China under the Grants No.90203005 and No.10475037, and the Natural Science Foundation of the Liaoning Scientific Committee(20032101).

\section{References}

[1] S. Fukuda et al. [Super-Kamiokande Collaboration], Phys. Rev. Lett. 86, 5651(2001); 5656(2001); Q. R. Ahmad et al. [SNO Collaboration], Phys. Rev. Lett. 87, 071301(2001); 89, 011301(2002); 89, 011302(2002); R. Davis, Rev. Mod. Phys. 75, $985(2003)$.

[2] Y. Fukuda et al. [Super-Kamiokande Collaboration], Phys. Rev. Lett. 81, 1562(1998); 82, 2644(1999); 85, 3999(2000).

[3] K. Eguchi et al. [KamLAND Collaboration], Phys. Rev. Lett. 90, 021802(2003); M. H. Ahn et al. [K2K Collaboration], Phys. Rev. Lett. 90, 041801(2003).

[4] C. T. Hill and E. H. Simmons, Phys. Rept. 381, 235(2003), [Erratum -ibid, 390, $553(2004)]$. 
[5] G. Burdman, Phys. Rev. Lett. 83, 2888(1999); H.-J. He, C.-P. Yuan, Phys. Rev. Lett. 83, 28(1999); H.-J. He, S. Kanemura, C.-P. Yuan, Phys. Rev. Lett. 89, 101803(2002).

[6] Chong-Xing Yue, Hong-Jie Zong, Shun-Zhi Wang, Phys. Lett. B 575, 25(2003); Chong-Xing Yue, Dong-Qi Yu, Zheng-Jun Zong, Phys. Lett. B 591, 220(2004).

[7] Chong-Xing Yue et al., Phys. Lett. B 496, 93(2000); Xue-Lei Wang et al., Phys. Rev. D 66, 075009(2002); Xue-Lei Wang et al., Phys. Rev. D 69, 055002(2004); Xue-Lei Wang et al., Phys. Rev. D 68, 115003(2003).

[8] Chong-Xing Yue, Lan-Jun Liu, Dong-Qi Yu, Phys. Rev. D 68, 035002(2003).

[9] Chong-Xing Yue et al., J. Phys. G 23, 163(1997); Chong-Xing Yue et al., Phys. Rev. D 64, 095004(2001); Jun-Jie Cao, Zhao-Hua Xiong, Jin-Min Yang, Phys. Rev. D 67, 071701(2003); Chong-Xing Yue et al., Nucl. Phys. B 650, 290(2003); Jun-Jie Cao, Guo-Li Liu, Jin-Min Yang, hep-ph/0311166.

[10] Chong-Xing Yue, Yuan-Ben Dai, Qing-Jun Xu, Guo-Li liu, Phys. Lett. B 525, $301(2002)$.

[11] Chong-Xing Yue, Dong-Qi Yu, Lan-Jun Liu, Phys. Rev. D 69, 095003(2004); ChongXing Yue, Wei Wang, Feng Zhang, J. Phys. G 30, 1065(2004).

[12] M. L. Brooks et al. [MEGA Collaboration], Phys. Rev. Lett. 83, 1521(1999); K. Abe et al. [Belle Collaboration], Phys. Rev. Lett. 92, 171802(2004).

[13] M. Aoki, talk at 21st International Conference on Neutrino Physics and Astrophysics, Neutrino 2004, Paris, France, 14-19 June 2004.

[14] K. S. Babu and C. Kolda, Phys. Rev. Lett 89, 241802(2002); A. Dedes, J. R. Ellis, and M. Raidal, Phys. Lett. B 549, 159(2002); M. Sher, Phys. Rev. D 66, 057301(2002).

[15] S. K. Kang and K. Y. Lee, Phys. Lett. B 521, 61(2001); R. A. Diaz, R. Martinez, and J. A. Rodrignez, Phys. Rev.D 67, 075011(2003) ; J. L. Diaz-Cruz, R. Noriega- 
Papaqui, A.Rosado, Phys. Rev.D 69, 095002(2004); Yu-Feng Zhou, J. Phys.G 30, $783(2004)$

[16] J. G. Korner, A. Pilaftsis and K. Schilcher, Phys. Rev.D 47, 1080(1993); J. L. DiazCruz and J. J. Toscano, Phys. Rev.D 62, 116005(2000); A. Brignole and A. Rossi, Phys. Lett. B 566, 217(2003); A. Brignole and A. Rossi, Nucl. Phys. B 701, 3(2004); S. Kanemura et al., Phys. Lett. B 599, 83(2004); S. Atag, K. O. Ozansoy, Phys. Rev.D 70, 053001(2004); E. Arganda, A. M. Curiel, and M. J. Herrero, Phys. Rev. D 71, 035011(2005).

[17] C. T. Hill, Phys. Lett. B 345, 483(1995); K. D. Lane and E. Eichten, Phys. Lett. B 352, 382(1995); K. D. Lane, Phys. Lett. B 433, 96(1998); G. Cvetic, Rev. Mod. Phys. 71, 513(1999).

[18] D. Kominis, Phys. Lett. B 358, 312(1995); G. Buchalla et al., Phys. Rev. D 53, 5185(1996); G. Burdman and D. Kominis, Phys. Lett. B 403, 101(1997).

[19] W. Loinaz and T. Takeuchi, Phys. Rev. D 60, 015005(1999).

[20] Chong-Xing Yue, Yu-Ping Kuang, Xue-Lei Wang and Wei-Bin Li, Phys. Rev. D 62, $055005(2000)$.

[21] Chong-Xing Yue, Qing-Jun Xu, Guo-Li Liu and Jian-Tao Li, Phys. Rev. D 63, $115002(2001)$.

[22] M.Passera, J. Phys. G31, R75(2005).

[23] Chong-Xing Yue, Qing-Jun Xu, Guo-Li Liu, J. Phys. G 27, 1807(2001).

[24] V. Barger, M. S. Berger, J. F. Gunion, T. Han, Phys. Rev. Lett. 75, 1462(1995); Nucl. Phys. Proc. Suppl. 51A, 13(1996); Phys. Rep. 286, 1(1997); R. Casalbuoni et al., JHEP 9908, 011(1999); V. D. Barger et al., hep-ph/0110340; C. Blochinger et al., hep-ph/0202199; H. Fraas et al., Eur. Phys. J. C 29, 587(2003). 
[25] E. Eichten, K. D. Lane and J. Womersley, Phys. Rev. Lett. 80, 5489(1998); R. Casalbuoni, et al., hep-ph/9801243; K. D. Lane, hep-ph/9801385; C. T. Hill, hepph/9802216; B. A. Dobrescu, hep-ph/9802259; Chong-Xing Yue, Gong-Ru Lu, JianTao Li, J. Phys. G 27, 913(2001).

[26] D. Atwood, L. Reina and A. Soni, Phys. Rev. Lett. 75, 3800(1995); M. Sher, Phys. Lett. B487, 151(2000); U. Cotti, M. Pineda, and G. Tavares-Velasco, hep-ph/0501162.

[27] Chong-Xing Yue, Lan-Jun Liu, Dong-Qi Yu, Phys. Rev. D 68, 035002(2003).

[28] R. S. Chivukula and E. H. Simmons, Phys. Rev. D 66, 015006(2002).

[29] T. Abe et al., [American Linear Collider Group], hep-ex/0106057; J. A. AguilarSaavedra et al., [ECFA/DESY LC Physics Working Group], hep-ph/0106315; K. Abe et al., [ACFA Linear Collider Working Group], hep-ph/0109166; G. Laow et al., ILC Techinical Review Committee, second report, 2003, SLAC-R-606.

[30] E. Boos et al., Nucl. Instrum. Meth. A472, 100(2001); B. Badelek et al., [ECFA/DESY Photon Collider Working Group], hep-ex/0108012; S. J. Brodsky, Intern. J. Mod. Phys. A 18, 2871(2003).

[31] S. J. Brodsky, T. Kinoshita and H. Terazawa, Phys. Rev. D 4, 1532(1971); H. Terazawa, Rev. Mod. Phys. 45, 615(1973); B. A. Kniehl, Phys. Lett.B 254, 267(1991).

[32] I. F. Ginzburg et al., Nucl. Instrum. Meth. in Phys. Res. 219, 5(1984); V. I. Telnov, Nucl. Instrum. Meth. 294, 72(1990). 